\author{
Miguel H. Hirata \\ Emeritus Member, ABCM \\ hirata@superonda.com.br \\ FAT/UERJ, Campos Regional de Resende, \\ 27537-000 Resende, RJ, Brazil \\ Luiz Antonio A. Pereira \\ Member, $A B C M$ \\ luizantp@unifei.edu.br \\ UNIFEI \\ Instituto de Engenharia Mecânica \\ 37500-903 Itajubá, MG, Brazil \\ Jan N. Recicar \\ jan.recicar@uninove.br \\ Washington $\mathrm{H}$. de Moura \\ whmoura@uninove.br \\ UNINOVE \\ Departamento de Ciências Exatas \\ 02117-010 São Paulo, SP, Brazil
}

\section{High Reynolds Number Oscillations of a Circular Cylinder}

This paper concerns the numerical simulation of the flow around an oscillating circular cylinder, which moves with constant velocity in a quiescent Newtonian fluid with constant properties. For each time step of the simulation a number of discrete Lamb vortices is placed close to the body surface; the intensity of each of these is determined such as to satisfy the no-slip boundary condition. The aerodynamic loads acting on the surface of cylinder are computed using the integral formulation derived from the pressure Poisson equation. The influence of the frequency and amplitude oscillation on the aerodynamic loads and on the Strouhal number is presented and discussed.

Keywords: heaving cylinder, aerodynamic loads, vortex shedding frequency, vortex method

\section{Introduction}

The analysis of the flow around an oscillating body in a quiescent fluid is of great technological importance and raises a vivid interest among the researches.

In fact, the structural elements and the equipments of an offshore platform used for oil exploitation oscillate due to the ocean wave action; the onshore structures such as towers, chimney stacks, bridge decks, etc. are subject to the wind action which induces to oscillatory motions, and the heat exchanger tubes vibrate as a result of the vortex shedding, etc.

The analysis and the comprehension of the complex phenomena that are present in the flow around an oscillating bluff body fascinate and have been challenging researchers for many decades. The oscillatory motion of small amplitude mainly modifies the near field flow changing its characteristics such as the flow regime inside the boundary layer, the pressure field, to mention the most important ones. On the other hand, the large amplitude oscillatory motion interacts with the far field wake with important consequences. Furthermore, the frequency and amplitude of the body oscillation define the frequency of vortex shedding which dictates the lift and drag coefficients behavior. Wake dynamics, unsteady phenomena associated with bluff body flows, the mechanisms that led to the formation and release of the vortex structures in the wake are a few of many aspects that deserve attention. For these reasons the literature is plenty of materials on the subject; these are the results of experimental and analytical works and more recently of numerical simulations; comprehensive reviews can be found in Sarpkaya (1979), Bearman (1984), Blevins (1990) and Williamson and Govardhan (2004). The experimental work of Williamson and Roshko (1988) deals with the synchronization regions and identifies many modes and vortex wake patterns with detailed explanations and descriptions; due to the difficulties in the experimental visualizations the Reynolds number was kept below 600. Dalton and Chantranuvatana (1980) present the average pressure distribution measured on the surface of oscillating cylinders and thus explaining some features of the near field flow. Blackburn and Henderson (1999) in their numerical simulations concentrated their attention to the flow at $\mathrm{Re}=500$ and the amplitude ratio 0.25 in order to study the effect of variations in frequency ratio on the entrainment phenomena produced by forced cross-flow oscillation within the primary synchronization regime. The work of Meneghini and Bearman (1995) deals with the numerical simulations of the flow past over an oscillating cylinder; they used a hybrid vortex method in their simulations and kept the Reynolds number with a low value.

To analyze the flow around an oscillating body one can hold the body fixed and let the whole mass of fluid oscillate or let the body oscillate in a calm fluid. This work utilizes the second approach to simulate numerically the fluid flow around a circular cylinder. Therefore, far away from the body the fluid is at rest except, perhaps, inside the viscous wake which is of importance when the body is bluff. For the present analysis two reference frames are used; the body fixed frame oscillates with respect to the inertial frame of reference.

For large amplitude motions the body boundary conditions are satisfied on the actual body surface at all time. If the amplitude of oscillation is small, to the first approximation, the body boundary conditions can be satisfied on the mean position of the body surface thus saving precious computational efforts, see Silva (2004).

A Lagrangian Vortex Method (VM) is used for the numerical simulations; fundamentals aspects of this method can be found in Leonard (1980), Sarpkaya (1989), Kamemoto (1994), Lewis (1999), Cottet and Koumoutsakos (2000), Stock (2007). The VM utilizes a cloud of free vortices to simulate the vorticity in the fluid region; each of these vortices is followed during the numerical simulation in a Lagrangian context, hence no spatial discretization is necessary as for example when working with the Eulerian methods.

The presented results are restricted to the heaving circular cylinder that moves with constant velocity $U$. In the numerical simulation, the Reynolds number based on the uniform flow and the cylinder diameter was kept constant and equal to $10^{5}$; even with such a high value, no attempt for turbulence modeling was made, see Alcântara Pereira et al. (2002). 
In the flow simulation around a fixed cylinder the vortex shedding frequency (which is obtained using the lift coefficient plot) is constant and, for reference, it is assumed that the Strouhal number takes the value 0.20 , with $10 \%$ uncertainty (Blevins, 1984).

For low amplitude cylinder oscillations the body boundary conditions are transferred from the actual position to the mean cylinder position thus leading to a simplified version of the methodology; numerical results show that this approach is valid if the amplitude ratio is less than 0.4 (Moura, 2007).

The full version of the methodology is used here to simulate the flow around a heaving circular cylinder. The numerical simulations show that there are three characteristic bands for the body oscillating frequency. Band I, for which $\mathrm{f}_{\mathrm{b}} \rightarrow 0$, the body oscillation has little influence and the vortex shedding frequency is almost independent of the body oscillating frequency. Band II represents a transition band in which the lock-in is partial and one can identify simulation periods in which lock-in is observed followed by simulation periods where this does not occur. Finally in the uppermost Band III the inertial components dominating the lock-in are always observed. The numerical findings show that the lock-in frequency depends on the amplitude of the body oscillation; in fact it decreases linearly with it in accordance to the low Reynolds number findings of Williamson and Roshko (1988).

Results for a rotating cylinder are presented elsewhere (Mustto et al., 1998).

The analysis of the near field flow illustrates the mechanism of vortex shedding when the body oscillates; this is not quite the same as the one found when the cylinder is fixed as the instantaneous pressure distributions on the cylinder surface and the instantaneous near field flow patterns show.

\section{Nomenclature}

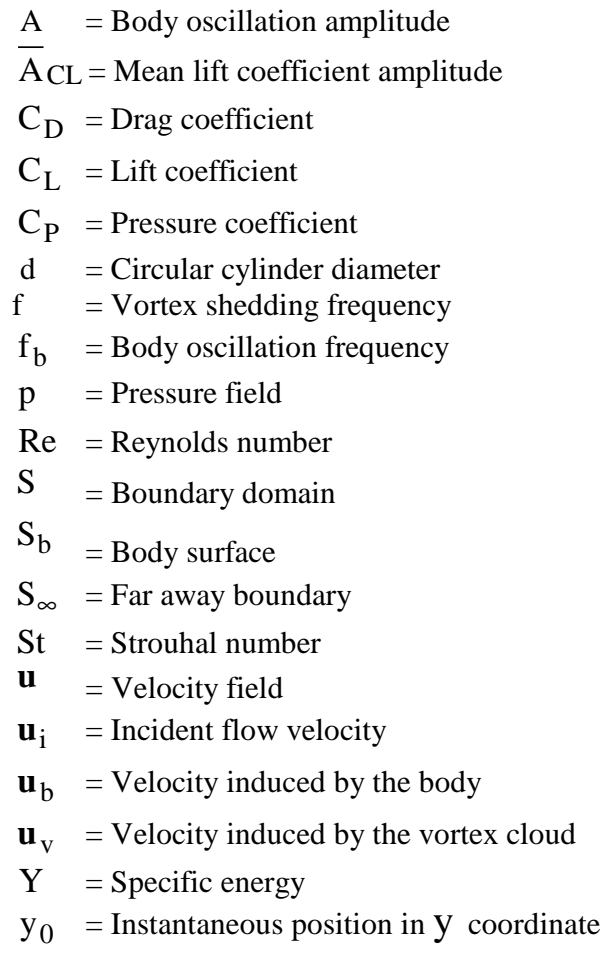

\section{Greek Symbols}

$$
\begin{array}{ll}
\omega & =\text { Angular velocity } \\
v & =\text { Kinematic viscocity } \\
\mu & =\text { Absolute viscosity } \\
\gamma & =\text { Vorticity distribution }
\end{array}
$$

$$
\begin{array}{ll}
\Gamma & =\text { Vortex strenght } \\
\omega & =\text { Vorticity field } \\
\Omega & =\text { Fluid domain } \\
\tau & =\text { Coordinate tangent to body surface } \\
\mathrm{n} & =\text { Coordinate normal to body surface } \\
\infty & =\text { Infinity }
\end{array}
$$

\section{Problem Definition}

The body surface is defined in the $(\xi, \eta)$ coordinate system by a scalar function $\mathrm{F}_{\mathrm{b}}(\xi, \eta)=0$, and for a circular cylinder $\mathrm{F}_{\mathrm{b}}$ is written as:

$$
\mathrm{F}_{\mathrm{b}}(\xi, \eta)=\xi^{2}+\eta^{2}-\mathrm{R}_{\mathrm{o}}^{2}=0
$$

Attention is restricted to a body motion with constant speed U, to which a heaving oscillatory motion defined by:

$$
\mathrm{y}_{\mathrm{o}}=\mathrm{A} \cos (\omega \mathrm{t})=\mathrm{A} \cos \left(2 \pi \mathrm{f}_{\mathrm{b}} \mathrm{t}\right)
$$

is superimposed, as shown in Fig. 1. The $(\mathrm{x}, \mathrm{y})$ is an inertial coordinate system.

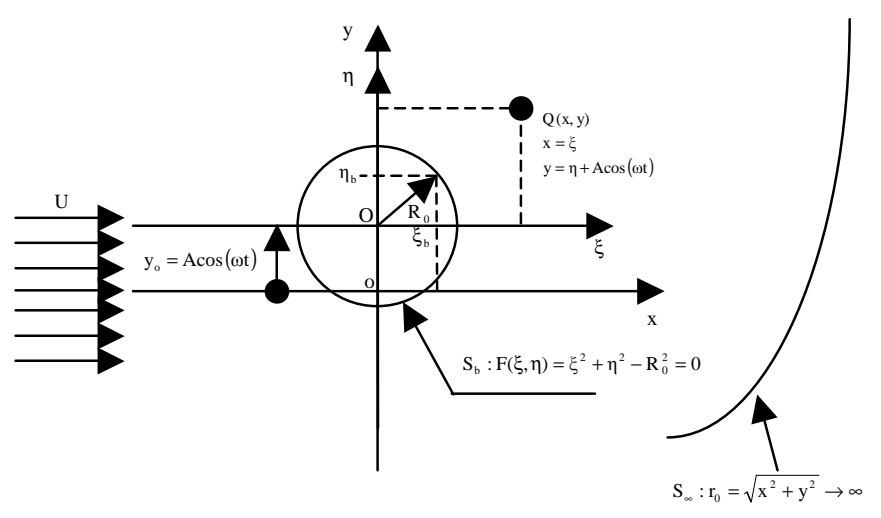

Figure 1. Definitions.

It is assumed that the flow of the Newtonian fluid with constant properties is incompressible and takes place in $\Omega$, which is defined by the surface $\mathrm{S}$ such that:

$$
\mathrm{S}=\mathrm{S}_{\mathrm{b}} \cup \mathrm{S}_{\infty}
$$

The fluid flow is governed by the non-dimensional equations:

$$
\begin{aligned}
& \frac{\partial u_{i}}{\partial x_{i}}=0, \quad i=1,2 \\
& \frac{D u_{i}}{D t}=-\frac{\partial p}{\partial x_{i}}+\frac{1}{\operatorname{Re}} \frac{\partial^{2} u_{i}}{\partial x_{j} \partial x_{j}}, \quad i=j=1,2
\end{aligned}
$$

The Reynolds number is defined by a characteristic body length $\mathrm{d}=2 \mathrm{R}_{\mathrm{o}}$ and the incoming flow velocity $\mathrm{U}$. The dimensionless time is defined as $\mathrm{d} / \mathrm{U}$. In addition, on $\mathrm{S}_{\mathrm{c}}$, the following boundary conditions are required:

$$
\mathrm{u}_{\mathrm{n}}=\mathrm{v}_{\mathrm{n}} \text { and } \mathrm{u}_{\tau}=\mathrm{v}_{\tau} \quad \text { on } \mathrm{S}_{\mathrm{b}}
$$


where $u_{n}$ and $u_{\tau}$ are the normal and tangential fluid particle velocity components and $\mathrm{v}_{\mathrm{n}}$ and $\mathrm{v}_{\tau}$ the body surface velocity components. Far away from the body, i.e., on $S_{\infty}$, the perturbation caused by the body motion fades, such that:

$$
|\mathbf{u}| \rightarrow 1
$$

\section{Numerical Method}

In many situations it is more natural to look at the curl of the velocity field rather than the more usual pressure-velocity form. This leads to the vorticity form of the Navier-Stokes equations, the vorticity transport equation, which presents no pressure term; in two dimensions it reads:

$$
\frac{\partial \omega}{\partial t}+u_{i} \frac{\partial \omega}{\partial x_{i}}=\frac{1}{\operatorname{Re}} \frac{\partial^{2} \omega}{\partial x_{i} \partial x_{i}}, \quad i=1,2
$$

where $\mathbf{u}(\mathbf{x}, t)$ is the velocity at position $\mathbf{x}$ and time $t$ and $\omega(\mathbf{x}, t)$ is the vorticity defined by:

$$
\omega \mathbf{k}=\nabla \times \mathbf{u},
$$

$\mathbf{k}$ being the unit vector perpendicular to the $(\mathrm{x}, \mathrm{y})-$ plane.

According to the Biot-Savart integral, one can recover the velocity as:

$$
\mathbf{u}=\mathbf{K} * \omega
$$

where $(*)$ indicates convolution and:

$$
\mathbf{u}(\mathbf{x}, \mathrm{t})=\int \mathbf{K}\left(\mathbf{x}-\mathbf{x}^{\prime}\right) \omega\left(\mathbf{x}^{\prime}, \mathrm{t}\right) \mathrm{d} \mathbf{x}^{\prime} .
$$

The spatially discretized vorticity field is represented by a cloud of point vortices, such that:

$$
\omega(\mathbf{x}, \mathrm{t})=\sum_{\mathrm{j}=1}^{\mathrm{NV}} \Gamma_{\mathrm{j}} \gamma\left(\mathbf{x}-\mathbf{x}_{\mathrm{j}}(\mathrm{t})\right)
$$

where $\gamma$ is the vorticity distribution of the jth vortex of the cloud and $\Gamma_{\mathrm{j}}$ its strength. If $\gamma$ is the Dirac delta function one recovers the potential vortex which has a singular velocity distribution. To overcome this unwanted behavior one may choose:

$$
\gamma(\mathbf{x})=\frac{1}{\mathrm{r}_{\mathrm{o}}^{\mathrm{n}}} \mathrm{f}\left(\frac{\mathbf{x}}{\mathrm{r}_{\mathrm{O}}}\right),
$$

f ( ) being radially symmetric and of compact support, $r_{o}$ is the cutoff radius and defines the support of $\gamma$. We use the Lamb vortex which has a Gaussian distribution with zero mean and a standard deviation of $\sqrt{2 v t}, v$ is the kinematic viscosity coefficient:

$$
\gamma(\mathbf{x})=\frac{1}{\pi r^{2}} \exp \left(-\frac{\mathbf{x}^{2}}{r_{0}^{2}}\right)
$$

\section{The Vortex Method}

In the Vortex Method the continuous vorticity field is represented by a cloud of discrete vortex blobs, Eq. (12). With this approach one reduces the computing effort to the calculation of the motion of these particles in order to satisfy the vorticity transport equation; this is performed in a Lagrangian context.

\section{Operator Splitting (Chorin, 1973)}

To calculate the particles motion, the operator splitting technique is used. The time integration is divided into two fractional steps: pure diffusion and convection.

Thus, during each time step, the linear diffusion equation:

$$
\frac{\partial \omega}{\partial t}=\frac{1}{\operatorname{Re}} \frac{\partial^{2} \omega}{\partial x_{i} \partial x_{i}}, \quad i=1,2
$$

and the non-linear convection equation:

$$
\frac{\partial \omega}{\partial t}=-u_{i} \frac{\partial \omega}{\partial x_{i}}, \quad i=1,2
$$

are solved independently.

\section{Convection of Vorticity}

Once the vorticity field is modeled by a cloud of discrete vortices, the convection equation (16a) is written in Lagrangian form as:

$$
\frac{\mathrm{d} \mathbf{x}_{\mathrm{j}}}{\mathrm{dt}}=\mathbf{u}_{\mathrm{j}}(\mathbf{x}, \mathrm{t}), \quad \mathrm{j}=1, \mathrm{NV}
$$

where NV is the number of point vortices in the cloud and the velocity field $\mathbf{u}(\mathbf{x}, \mathbf{t})$ can be split in three parts, Alcântara Pereira et al. (2003):

$$
\mathbf{u}(\mathbf{x}, \mathrm{t})=\mathbf{u i}(\mathbf{x}, \mathrm{t})+\mathbf{u b}(\mathbf{x}, \mathrm{t})+\mathbf{u v}(\mathbf{x}, \mathrm{t})
$$

The contribution of the incident flow is represented by $\mathbf{u i}(\mathbf{x}, \mathrm{t})$. For an uniform oncoming flow its components take the form:

$$
\mathrm{ui}_{1}=1 \text { and } \quad \mathrm{ui}_{2}=0
$$

The body contributes with $\mathbf{u b}(\mathbf{x}, \mathbf{t})$, which can be obtained, for example, using the Boundary Element Method. The two components can be written as:

$$
\mathrm{ub}_{\mathrm{i}}(\xi, \mathrm{t})=\sum_{\mathrm{j}=1}^{\mathrm{NP}} \sigma_{\mathrm{j}} \mathrm{G}_{\mathrm{ij}}\left(\xi-\xi_{\mathrm{j}}(\mathrm{t})\right), \quad \mathrm{i}=1,2 \quad \text { and } \mathrm{i} \neq \mathrm{j}
$$

where $\mathrm{G}_{\mathrm{ij}}\left(\xi-\xi_{\mathrm{j}}(\mathrm{t})\right)$ is an appropriated defined kernel, see Katz and Plotkin (1991). In this paper, sources with constant density, $\sigma_{k}$, were distributed over NP flat panels representing the body surface. At this point it is worth to observe that the panels (the body surface) are oscillating with respect to the inertial frame of reference; therefore the body contribution in the inertial frame of reference is (Recicar et al., 2006):

$$
\mathrm{u}(\mathbf{x}, \mathbf{y}, \mathrm{t})=\mathrm{u}(\xi, \eta, \mathrm{t})
$$




$$
\mathrm{v}(\mathbf{x}, \mathbf{y}, \mathrm{t})=\mathrm{v}(\xi, \eta, \mathrm{t})+\dot{\mathrm{y}}_{\mathrm{o}}(\mathrm{t})
$$

Finally the velocity $\mathbf{u v}(\mathbf{x}, \mathrm{t})$, due to the vortex interactions has its components written as:

$$
u v_{i}(x, t)=\sum_{j=1}^{N V} \Gamma_{j} c_{i j}\left(x-x_{j}(t)\right)
$$

where $\Gamma_{\mathrm{j}}$ is the $\mathrm{j}$-vortex strength and $\mathrm{c}_{\mathrm{ij}}\left(\mathbf{x}-\mathbf{x}_{\mathrm{j}}(\mathrm{t})\right)$ is the $\mathrm{i}$ component of the velocity induced, at point $\mathbf{x}$, by a unit strength vortex located at $\mathrm{x}_{\mathrm{j}}(\mathrm{t})$. We use Lamb vortex, therefore:

$$
\mathrm{u}_{\theta}^{\mathrm{kj}}=-\frac{\Gamma_{\mathrm{j}}}{2 \pi} \frac{1}{\mathrm{r}_{\mathrm{kj}}}\left[1-\exp \left(-5,02572 \frac{\mathrm{r}_{\mathrm{kj}}^{2}}{\sigma_{0}^{2}}\right)\right]
$$

Euler expression represents the first order approximation to the solution of the Eq. (16b):

$$
\mathrm{x}_{\mathrm{i}}(\mathrm{t}+\Delta \mathrm{t})=\mathrm{x}_{\mathrm{i}}(\mathrm{t})+\mathrm{u}_{\mathrm{i}}(\mathbf{x}, \mathrm{t}) \Delta \mathrm{t} \quad \mathrm{i}=1,2
$$

\section{Diffusion of Vorticity}

The solution to the diffusion Eq. (15) in the Lagrangian context is obtained using the Random Walk Method (Lewis, 1999) which aims to simulate the Brownian motion of the particles. According to this method, the random walk diffusion displacement $\boldsymbol{\eta}(\mathbf{x}, \mathbf{t})$, with a zero mean and a $(2 \Delta t / R e)$ variance, is added after the convective step. Hence the position of each vortex, at the instant $(t+\Delta t)$ is given by:

$$
\mathrm{x}_{\mathrm{i}}(\mathrm{t}+\Delta \mathrm{t})=\mathrm{x}_{\mathrm{i}}(\mathrm{t})+\mathrm{u}_{\mathrm{i}}(\mathrm{x}, \mathrm{t}) \Delta \mathrm{t}+\eta_{\mathrm{i}} \quad \mathrm{i}=1,2
$$

The random displacement components are:

$$
\begin{aligned}
& \mathrm{x}_{\mathrm{d}}^{(\mathrm{i})}=\sqrt{\frac{4 \Delta \mathrm{t}}{\operatorname{Re}} \ln \left(\frac{1}{\mathrm{P}}\right)}[\cos (2 \pi \mathrm{Q})] \\
& \mathrm{y}_{\mathrm{d}}^{(\mathrm{i})}=\sqrt{\frac{4 \Delta \mathrm{t}}{\operatorname{Re}} \ln \left(\frac{1}{\mathrm{P}}\right)}[\sin (2 \pi \mathrm{Q})]
\end{aligned}
$$

with $\mathrm{P}$ and $\mathrm{Q}$ being random numbers laying between zero and one.

\section{Aerodynamic Loads}

Once determined the vorticity field, the pressure calculation starts with the Bernoulli function, defined by Uhlman (1992) as

$$
\mathrm{Y}=\mathrm{p}+\frac{\mathrm{u}^{2}}{2}, \mathrm{u}=|\mathbf{u}|
$$

Kamemoto (1993) used the same function and starting from the Navier-Stokes equations was able to write a Poisson equation for the pressure. This equation was solved using a finite difference scheme. Here the same Poisson equation was derived and its solution was obtained through the following integral formulation (Shintani and Akamatsu, (1994):

$$
\mathrm{H} \overline{\mathrm{Y}_{\mathrm{i}}}-\int_{\mathrm{S}_{1}} \overline{\mathrm{Y}} \nabla \mathrm{G}_{\mathrm{i}} \cdot \mathbf{e}_{\mathrm{n}} \mathrm{d} \mathrm{S}=\iint_{\Omega} \nabla \mathrm{G}_{\mathrm{i}} \cdot(\mathbf{u} \times \boldsymbol{\omega}) \mathrm{d} \Omega-\frac{1}{\mathrm{Re}} \int_{\mathrm{S}_{1}}\left(\nabla \mathrm{G}_{\mathrm{i}} \times \boldsymbol{\omega}\right) \cdot \mathbf{e}_{\mathrm{n}} \mathrm{dS}
$$

where $\mathrm{H}=1$ in the fluid domain, $\mathrm{H}=0.5$ on the boundaries and $\mathrm{G}$ is a fundamental solution of the Laplace equation, Alcântara Pereira $e t$ al. (2004).

The drag and lift coefficients can be expressed by (Ricci, 2002):

$$
\begin{aligned}
& C_{D}=-\sum_{j=1}^{N P} 2\left(p_{j}-p_{\infty}\right) \Delta S_{j} \sin \theta_{j}=-\sum_{j=1}^{N P} C_{P} \Delta S_{j} \sin \theta_{j} \\
& C_{L}=-\sum_{j=1}^{N P} 2\left(p_{j}-p_{\infty}\right) \Delta S_{j} \cos \theta_{j}=-\sum_{j=1}^{N P} C_{P} \Delta S_{j} \cos \theta_{j}
\end{aligned}
$$

\section{Simulations and Results}

The attention is restricted to the analysis of the flow around a circular cylinder which is moving steadily in an otherwise quiescent fluid; to this uniform flow a heaving motion is superimposed:

$$
\mathrm{y}_{\mathrm{o}}=\mathrm{A} \cos (\omega \mathrm{t})=\mathrm{A} \cos \left(2 \pi \mathrm{f}_{\mathrm{b}} \mathrm{t}\right)
$$

In the calculations the cylinder surface is represented by 100 flat panels on which sources of constant density are distributed; the time step is taken as $\Delta t=0.05$. In each time step the nascent vortices were placed into the cloud through a displacement $\varepsilon=\sigma_{0}=0.0032 \mathrm{~d}$ normal to the panels. The aerodynamic loads computations starts at $\mathrm{t}=10$.

In the numerical simulation the Reynolds number based on the uniform flow and the cylinder diameter were kept constant and equal to $10^{5}$.

\section{Flow around a Fixed Circular Cylinder}

As an introduction to the analysis of the flow around a cylinder, some general results for a fixed cylinder are presented.

Figure 2 shows the evolution of the lift and drag coefficients as the numerical simulations proceed. As expected, the lift coefficient oscillates around the mean value (zero) and with a frequency - $\mathrm{f}-$ which is the vortex shedding frequency; the drag coefficient curve oscillates with a frequency that is twice this value $(2 \mathrm{f})$. The mean amplitude of the lift coefficient curve is indicated by $\overline{A_{C L}}$.

The Strouhal number is defined as:

$$
S_{t}=\frac{f d}{U}
$$

Mean values of the Strouhal number, lift and drag coefficient are presented in Table 1 and they show good agreement with data available in the literature. As mentioned in the introduction section it is assumed that the Strouhal number for a fixed cylinder takes the value 0.20 for future references.

The analysis of the flow shows that the largest value of the lift coefficient is attained when a cluster of free vortices leaves the body surface $\left(\theta \sim 100^{\circ}\right)$ toward the viscous wake; this cluster rotates in the clockwise direction (Fig. 3a). The lowest value of this coefficient is observed when another cluster, now rotating in the anti-clockwise direction (Fig. 3b), leaves the body surface $\left(\theta \sim 260^{\circ}\right)$. 


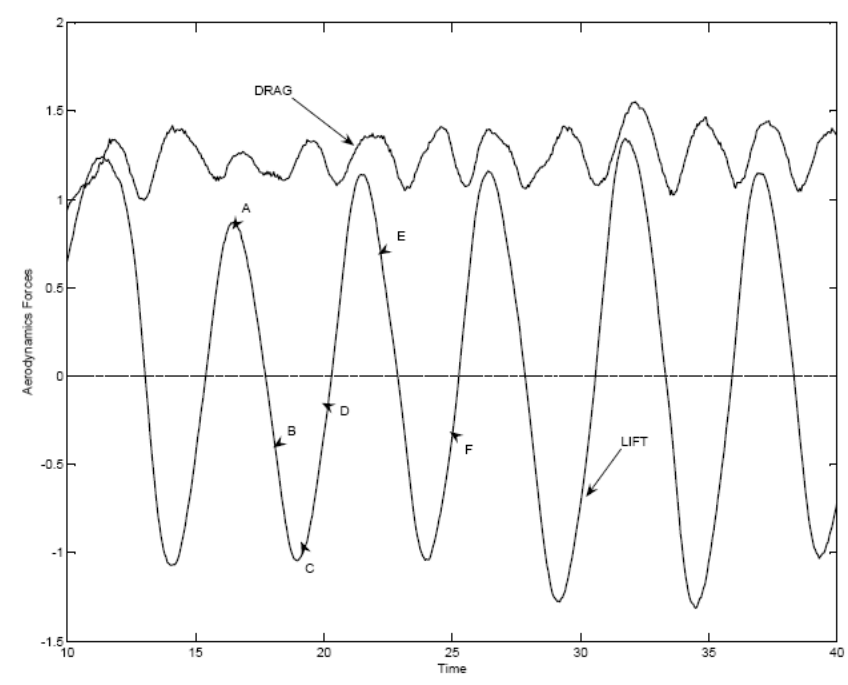

Figure 2. Lift and drag coefficients for fixed circular cylinder.

Table 1. Mean lift and drag coefficient for fixed circular cylinder.

\begin{tabular}{|c|c|c|c|c|}
\hline $\mathrm{Re}=10^{5}, \mathrm{~A}=0, \omega=0$ & $\overline{\mathrm{C}_{\mathrm{L}}}$ & $\overline{\mathrm{C}_{\mathrm{D}}}$ & $\mathrm{St}$ & $\overline{\mathrm{A}}_{\mathrm{CL}}$ \\
\hline Blevins (1984) & --- & 1,20 & 0,19 & --- \\
\hline Mustto et al . (1998) & --- & 1,22 & 0,22 & --- \\
\hline Alcântara Pereira et al . (2002) & 0,04 & 1,21 & 0,22 & --- \\
\hline Silva (2004) & $-0,03$ & 1,23 & 0,20 & --- \\
\hline Present Simulation & 0,07 & 1,22 & 0,20 & 1,058 \\
\hline
\end{tabular}

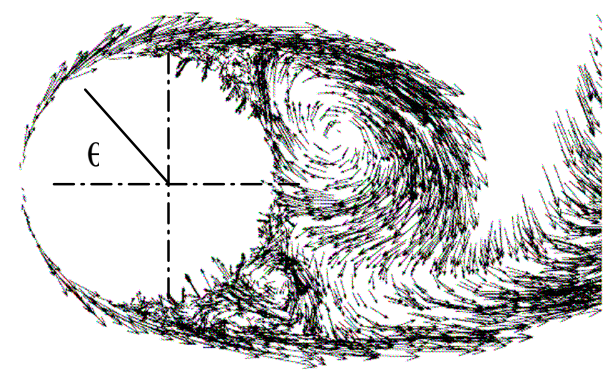

(a) $\mathrm{t}=16.5$

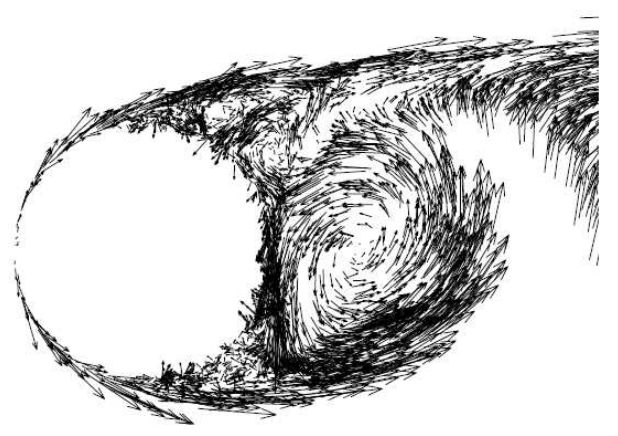

(b) $\mathrm{t}=19,4$

Figure 3. Near wake behavior for fixed circular cylinder.
Figure 4 presents mean pressure values on the body surface. One can observe a good agreement with data available in the literature, except in a small neighborhood of $\theta \sim 80^{\circ}$.

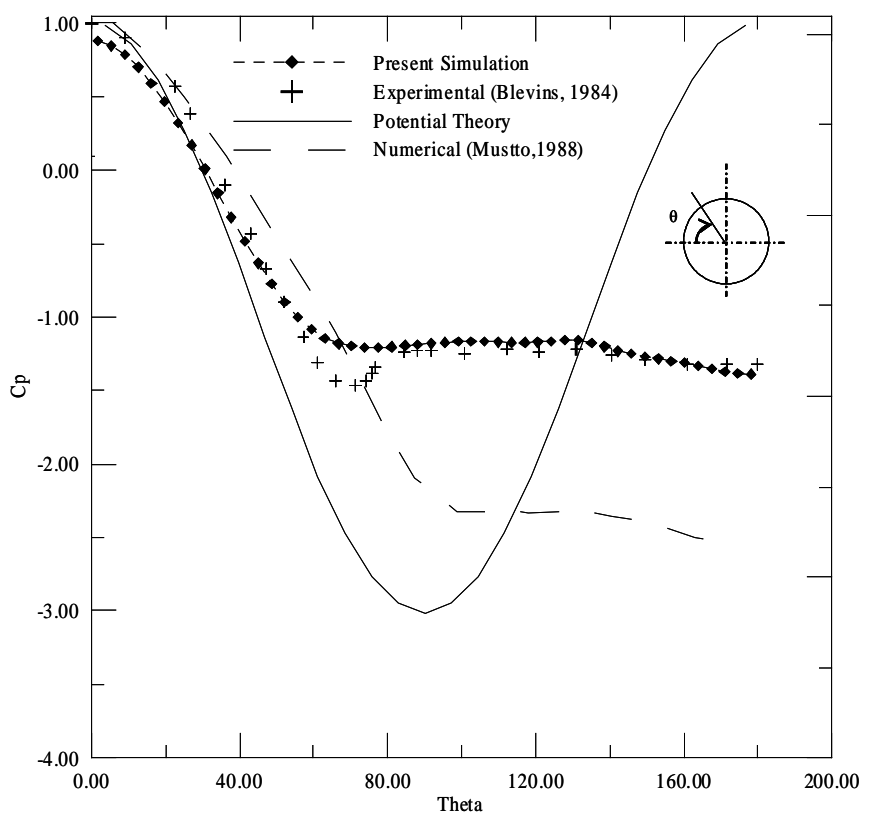

Figure 4. Mean pressure coefficient values for fixed circular cylinder.

The instantaneous pressure distributions are presented in Fig. 5. Distribution A, B, C, D, E and F are related respectively to points A, B, C, D, E and F found in Fig. 2.

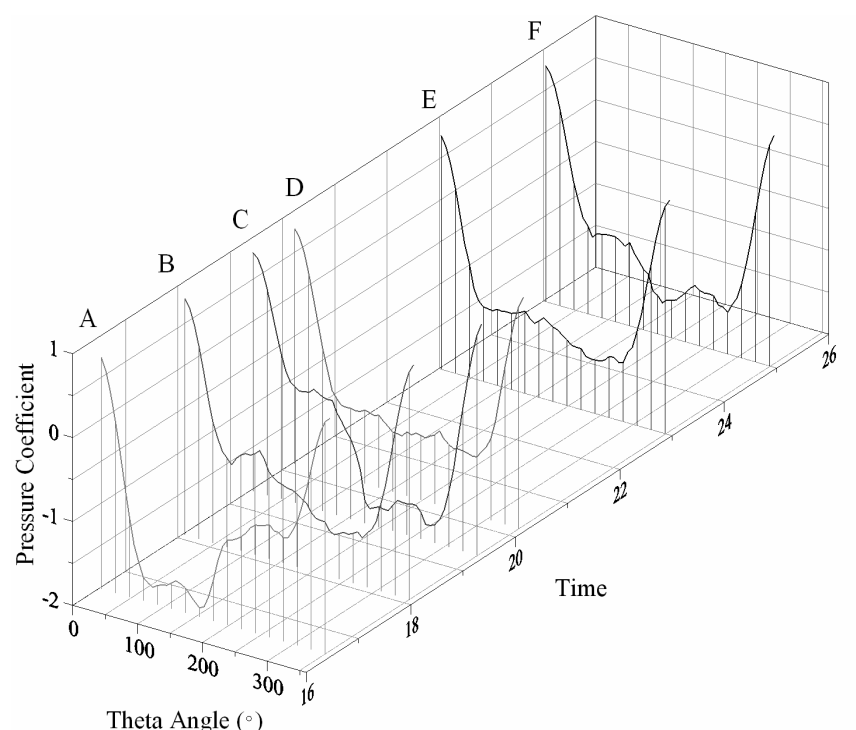

Figure 5. Instantaneous pressure distribution on the surface of a fixed circular cylinder.

\section{Flow around a Cylinder Oscillating with Small Amplitude}

In heaving motion of small amplitude, $(\mathrm{A} / \mathrm{d})=\mathrm{O}(\varepsilon), \varepsilon \rightarrow 0$, an important simplification is possible. In fact, discarding higher order terms in the Taylor expansion of the body boundary conditions, Eq. (6), one gets: 


$$
\begin{aligned}
& \mathrm{u}_{\mathrm{n}}=\mathrm{v}_{\mathrm{n}} \\
& \text { on } \overline{\mathrm{S}}_{\mathrm{b}} \\
& \mathrm{u}_{\tau}=\mathrm{v}_{\tau}
\end{aligned}
$$

where $\overline{\mathrm{S}}_{\mathrm{b}}$ is the mean position of the body surface with respect to the inertial frame of reference, as illustrated in Figure 6. The above expressions show that, to the first approximation, the body boundary conditions can be transferred from the actual to the mean body position. In order to take care of the motion induced by the oscillating movement, these modified boundary conditions possess an extra term that represents an additional source distribution on the body surface. As a consequence, the numerical simulation of the flow can proceed considering a steady body with modified body boundary conditions.

This observation leads to a considerable reduction in the computational effort which is very important in the analysis of many practical situations where small amplitude vibrations occur.

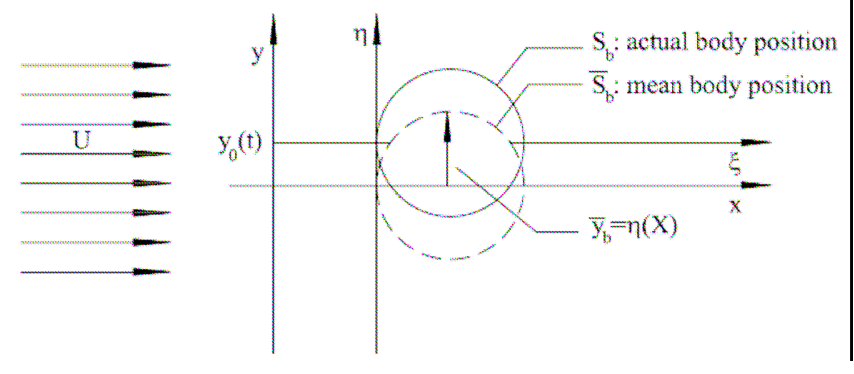

Figure 6. Small amplitude oscillations characteristics.

The results of these simulations where reported elsewhere, Silva (2004) and Moura et al.(2006). Here we present only a few values for comparisons, Table 2. From these values one can assume that the transferring of the body boundary conditions produce reasonable results up to $(\mathrm{A} / \mathrm{d})=0.3 \sim 0.4$.

\section{Flow around a Cylinder Oscillating with Large Amplitude}

For the analysis of the flow around a large amplitude heaving cylinder the body boundary conditions cannot be transferred from the actual position to the mean position.

The numerical simulations show that the flow is influenced mainly by the body oscillation amplitude, $\mathrm{A}$ and the frequency $\mathrm{f}_{\mathrm{b}}$. In fact one can identify three main bands of the body oscillation frequency.

Table 2. Mean lift and drag coefficients using the simplified approach.

\begin{tabular}{|c|c|c|c|c|c|c|c|c|c|}
\cline { 3 - 11 } & \multicolumn{4}{c|}{ Recicar (2007) } & \multicolumn{4}{c|}{ Moura (2007) } \\
\hline $\begin{array}{c}\text { Angular } \\
\text { Velocity } \\
(\omega)\end{array}$ & $\begin{array}{c}\text { Amplitude } \\
(\mathrm{A})\end{array}$ & $\overline{\mathrm{C}_{\mathrm{L}}}$ & $\overline{\mathrm{C}_{\mathrm{D}}}$ & $\mathrm{St}$ & $\mathrm{St}_{\mathrm{b}}$ & $\overline{\mathrm{C}_{\mathrm{L}}}$ & $\overline{\mathrm{C}_{\mathrm{D}}}$ & $\mathrm{St}$ & $\mathrm{St}_{\mathrm{b}}$ \\
\hline \multirow{5}{*}{0,05} & 0,01 & $-0,051$ & 1,233 & 0,196 & 0,008 & 0,027 & 1,215 & 0,206 & 0,008 \\
\cline { 2 - 12 } & 0,05 & 0,069 & 1,195 & 0,198 & 0,008 & 0,008 & 1,247 & 0,198 & 0,008 \\
\cline { 2 - 11 } & 0,1 & 0,071 & 1,184 & 0,207 & 0,008 & $-0,01$ & 1,24 & 0,215 & 0,008 \\
\cline { 2 - 11 } & 0,15 & 0,067 & 1,258 & 0,208 & 0,008 & 0,086 & 1,235 & 0,197 & 0,008 \\
\cline { 2 - 11 } & 0,2 & 0,124 & 1,185 & 0,193 & 0,008 & 0,092 & 1,256 & 0,199 & 0,008 \\
\cline { 2 - 10 } & 0,3 & 0,162 & 1,209 & 0,21 & 0,008 & 0,1313 & 1,2 & 0,209 & 0,008 \\
\cline { 2 - 10 } & 0,5 & 0,269 & 1,151 & 0,211 & 0,008 & 0,165 & 1,203 & 0,21 & 0,008 \\
\hline
\end{tabular}

At low frequency body oscillations, Band I, the flow behaves much like if the body were fixed and the amplitude of the cylinder oscillation has negligible influence on the vortex shedding frequency $f$. The Strouhal number assumes values that are very close to 0.20 , that is $S_{t}=O(0.2)$ as $f_{b} \rightarrow 0$.

In Band III the body oscillates at high frequencies, the body frequency of oscillation $f_{b}$ dominates the phenomena and the frequency of vortex shedding $\mathrm{f}$ is locked to it.

Finally, in Band II, the frequency of body oscillations lies in between the two other bands. Band II is a transition Band and one can eventually observe the locking of the frequencies.

Here the body Strouhal number is defined as

$$
\mathrm{St}_{\mathrm{b}}=\frac{\mathrm{f}_{\mathrm{b}} \mathrm{d}}{\mathrm{U}}
$$

and the wavelength ratio, as defined by Williamson and Roshko (1988), is:

$$
\frac{\lambda}{\mathrm{d}}=\frac{\mathrm{U}}{\mathrm{df}_{\mathrm{b}}}
$$

Figure 7 shows plots of the Strouhal number as a function of the body Strouhal number. The analysis of these figures shows that, in general, the high Reynolds number simulations agree quite well with the low Reynolds number vortex synchronization regions devised by Williamson and Roshko (1988), except that the synchronization begins at a lower value of $\mathrm{St}_{\mathrm{b}}$ (or higher value of the wavelength ratio), which is quite reasonable due to the increasing importance of the inertial effects as the Reynolds number assumes higher values.

Figure 8 utilizes the data from the previous figures to show the dependence of the lock on frequency on the amplitude of the body oscillations.

In Table 3 the body frequency of oscillation is kept constant with a low value, which corresponds to $\mathrm{St}_{\mathrm{b}}=0.008$, Band $\mathrm{I}$.

Table 3. Results for low frequency of oscillations.

\begin{tabular}{|c|c|c|c|c|c|c|}
\hline $\begin{array}{c}\text { Angular } \\
\text { Frequency } \\
(\omega)\end{array}$ & $\begin{array}{c}\text { Amplitude } \\
(\mathrm{A})\end{array}$ & $\overline{\mathrm{C}_{\mathrm{L}}}$ & $\overline{\mathrm{C}_{\mathrm{D}}}$ & $\mathrm{St}$ & $\mathrm{St}_{\mathrm{b}}$ & $\overline{\mathrm{A}_{\mathrm{CL}}}$ \\
\hline \multirow{7}{*}{0,05} & 0,01 & $-0,051$ & 1,233 & 0,196 & 0,008 & 1,171 \\
\cline { 2 - 7 } & 0,05 & 0,069 & 1,195 & 0,198 & 0,008 & 1,127 \\
\cline { 2 - 7 } & 0,1 & 0,071 & 1,184 & 0,207 & 0,008 & 1,001 \\
\cline { 2 - 7 } & 0,15 & 0,067 & 1,258 & 0,208 & 0,008 & 1,122 \\
\cline { 2 - 7 } & 0,2 & 0,124 & 1,185 & 0,192 & 0,008 & 1,070 \\
\cline { 2 - 7 } & 0,3 & 0,162 & 1,209 & 0,210 & 0,008 & 1,042 \\
\cline { 2 - 7 } & 0,5 & 0,269 & 1,151 & 0,211 & 0,008 & 0,971 \\
\hline
\end{tabular}

One observes that the Strouhal number is quite insensitive to the amplitude of the body oscillation and the amplitude of the lift coefficient curve seems to behave in the same manner without a definite pattern, as shown in Figure 9. 


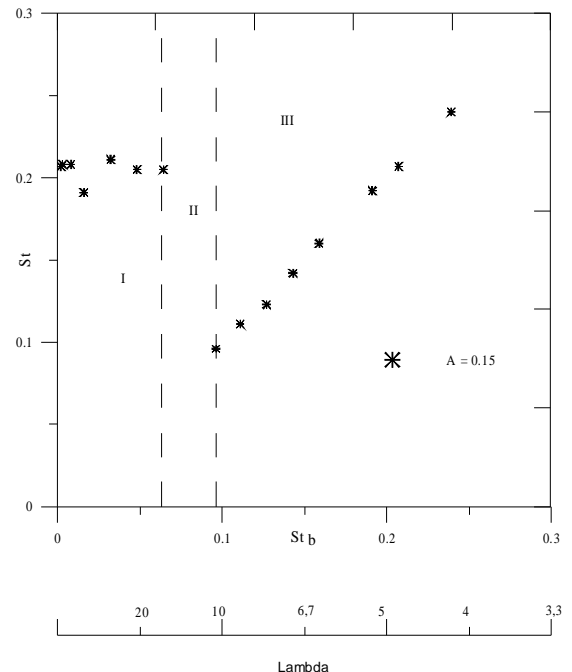

(a)

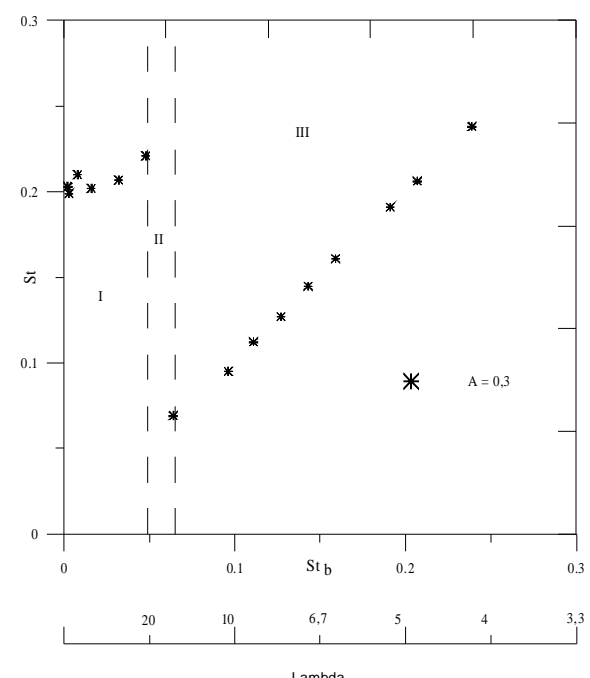

(b)

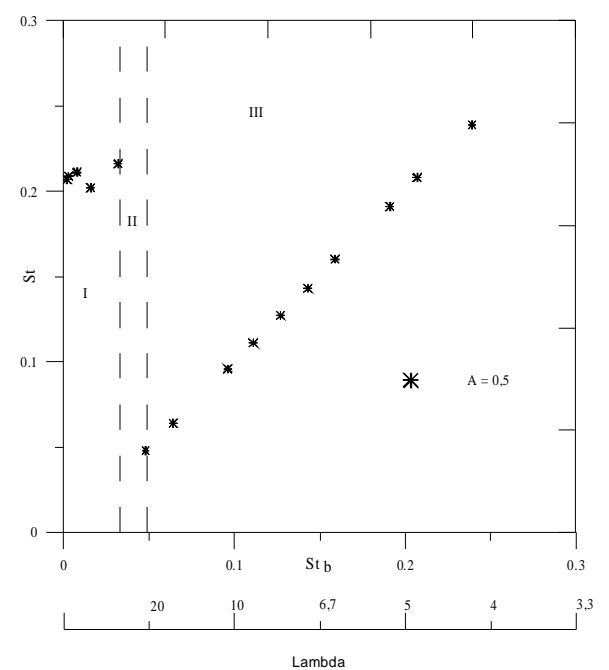

(c)

Figure 7. Frequency of vortex shedding: in (a) $A=0,15$, (b) $A=0,30$ and (c) $A=0,50$.

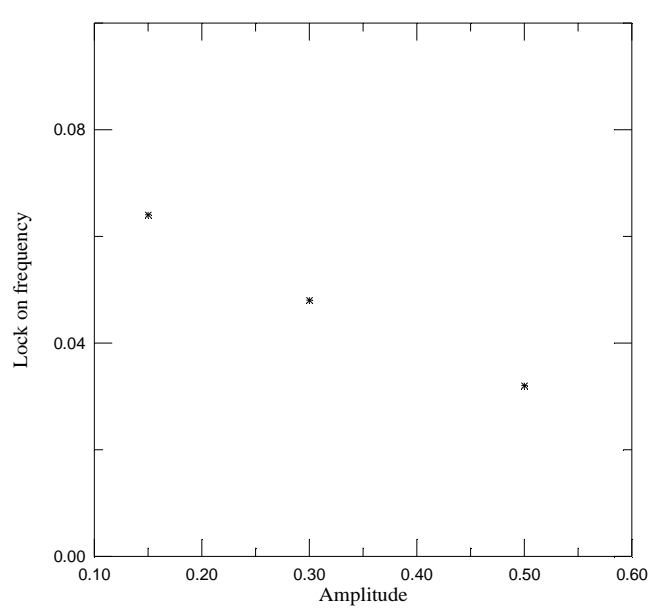

Figure 8. Lock on frequency.

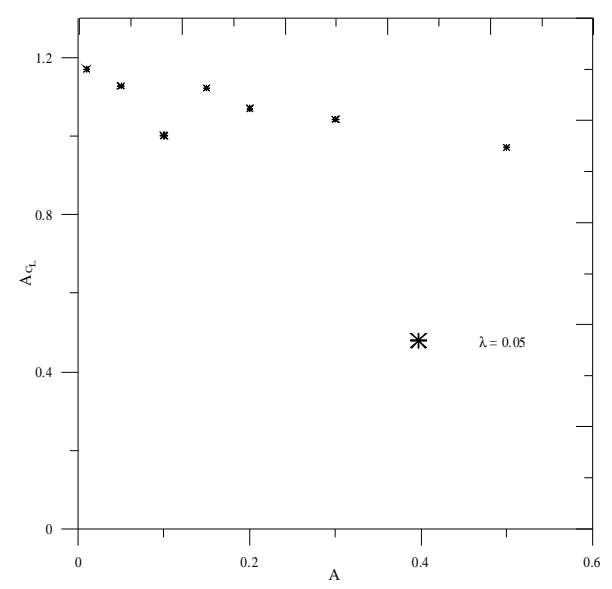

Figure 9. Lift coefficient amplitude for low frequency.

In Table 4 the body frequency of oscillation is kept constant with a high value which corresponds to $\mathrm{St}_{\mathrm{b}}=0.239$. As the amplitude of oscillation increases the synchronization of the frequencies is clearly observed and the drag coefficient decreases. The amplitude of the lift curve now has a definite behavior, increasing linearly as shown in Fig. 10.

Table 4. Results for high frequency of oscillations.

\begin{tabular}{|c|c|c|c|c|c|c|}
\hline $\begin{array}{c}\text { Angular } \\
\text { Frequency } \\
(\omega)\end{array}$ & $\begin{array}{c}\text { Amplitude } \\
(\mathrm{A})\end{array}$ & $\overline{\mathrm{C}_{\mathrm{L}}}$ & $\overline{\mathrm{C}_{\mathrm{D}}}$ & $\mathrm{St}$ & $\mathrm{St}_{\mathrm{b}}$ & $\overline{\mathrm{A}_{\mathrm{CL}}}$ \\
\hline \multirow{7}{*}{1,5} & 0,01 & 0,018 & 1,278 & 0,203 & 0,239 & 1,177 \\
\cline { 2 - 7 } & 0,05 & $-0,009$ & 1,095 & 0,240 & 0,239 & 1,094 \\
\cline { 2 - 7 } & 0,1 & 0,054 & 0,886 & 0,240 & 0,239 & 1,211 \\
\cline { 2 - 7 } & 0,15 & 0,023 & 0,631 & 0,240 & 0,239 & 1,234 \\
\cline { 2 - 7 } & 0,2 & 0,008 & 0,347 & 0,239 & 0,239 & 1,467 \\
\cline { 2 - 7 } & 0,3 & 0,044 & 0,172 & 0,238 & 0,239 & 1,805 \\
\cline { 2 - 7 } & 0,5 & 0,102 & $-0,14$ & 0,239 & 0,239 & 2,231 \\
\hline
\end{tabular}




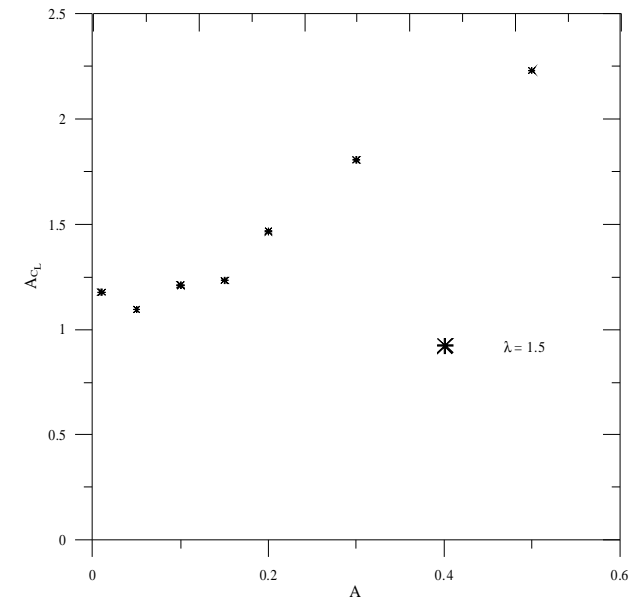

Figure 10. Lift coefficient amplitude for high frequency.

As done for a fixed cylinder, Figure 11 shows the lift and drag coefficient curves obtained as the numerical simulation proceeds; this figure refers to a condition where the synchronization of the frequencies is observed.

When the cylinder is at its highest position, point $\mathrm{A}$, the lift coefficient is close to its lowest value (highest negative value) which is consistent with the pressure distribution shown in Figure 12; in this figure a positive pressure distribution, on the upper surface, can clearly be observed; as expected just the opposite occur when the cylinder is at its lowest position, point $\mathrm{C}$.

Points B and D represent intermediates positions of the cylinder and it is interesting to note that the highest pressures are observed in front and at the rear of the cylinder, almost canceling each other which, by its turn, results in almost zero drag as predicted by the drag coefficient curve shown in Figure 11.

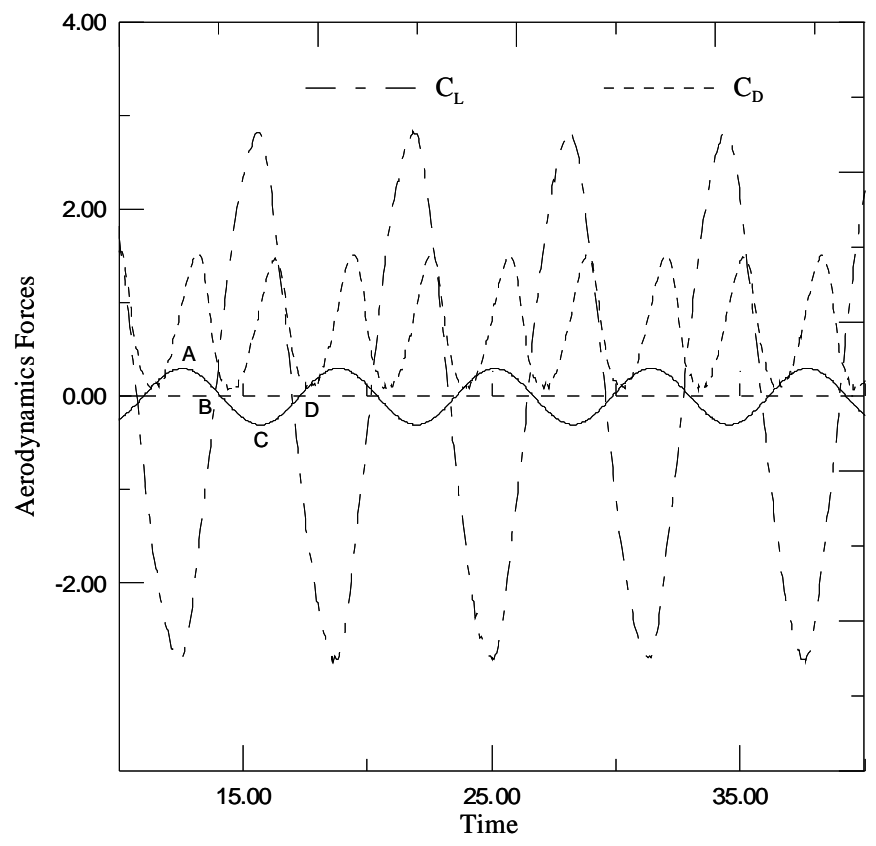

Figure 11. Lift and drag coefficients: $\omega=1$ and $A=0.3$.

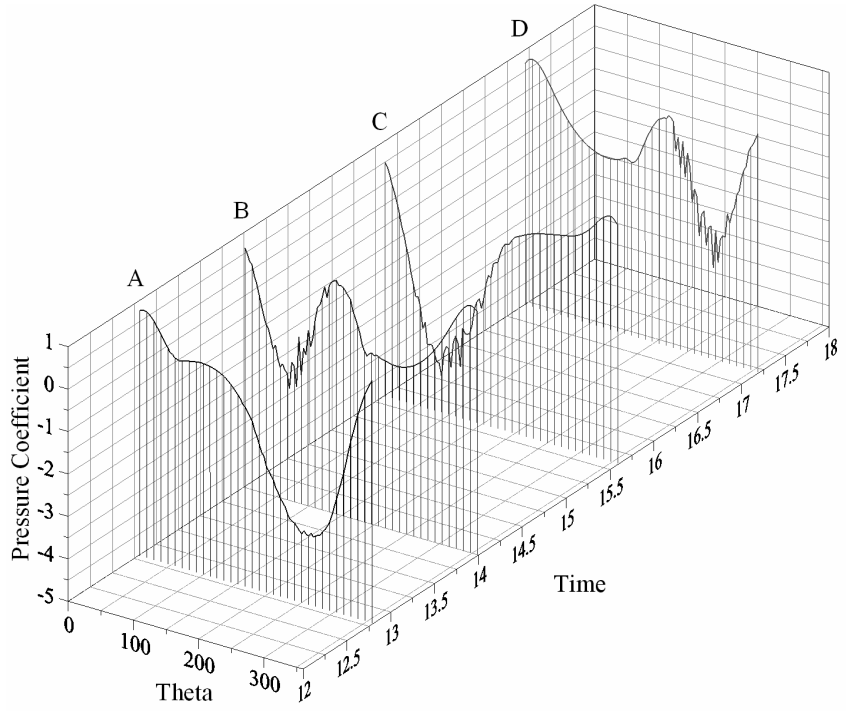

Figure 12. Instantaneous pressure distribution: $\omega=1$ and $A=0.3$.

\section{Conclusions and Summary}

The flow around a circular cylinder is analyzed using a numerical Lagrangian simulation, the Vortex Method. All the simulations were carried out with a high value of the Reynolds number; no attempt to use a turbulence modeling was made.

A general introduction to the method is presented and used for the simulation of the flow around a fixed cylinder, obtaining results which demonstrated good agreement with known values from the literature.

A simplified version of the methodology, valid for low amplitude oscillations, was also used to find out that $(\mathrm{A} / \mathrm{d})<(0.4 \sim 0.5)$ represents the limits of its applicability. This is an important result since it shows that the simplified version can be used in most of the situations where vibration problems occur (Moura, 2007). The understanding of several phenomena that occur due to the interaction between flowing fluid and heat exchangers and chimneys are typical examples.

The full version was used systematically to analyze the flow around a heaving cylinder. The (St versus $\mathrm{St}_{\mathrm{b}}$ ) plots show that one can identify three bands with clear characteristics behavior. In band I (low value of $\mathrm{St}_{\mathrm{b}}$ ) the flow behaves in much the same way as if the cylinder were fixed; St assumes values very close to 0.2 . Band II is a transition band. Finally in Band III (high value of $\mathrm{St}_{\mathrm{b}}$ ) inertial effects dominate and the shedding frequency is locked to the body oscillation frequency.

In Band III it is remarkable to notice that:

a) As the amplitude of body oscillation increases, the drag coefficient decreases systematically

b) As the amplitude of body oscillation increases, the amplitude of the lift coefficient curve increases linearly. Each band limit depends on body oscillation amplitude during numerical simulation.

\section{Acknowledgements}

This research was supported by the $\mathrm{CNPq}$ (Brazilian Research Agency) Proc. 47279/2004-8 and FAPEMIG (Research Foundation of the State of Minas Gerais) Proc. TEC-748/04. 


\section{References}

Alcântara Pereira, L.A., Ricci, J.E.R., Hirata, M.H. and Silveira Neto, A., 2002, "Simulation of Vortex-Shedding Flow about a Circular Cylinder with Turbulence Modelling", International Society of CFD / CFD Journal, $11 / 3$, pp. 315-322.

Alcântara Pereira, L.A., Hirata, M.H. and Silveira Neto, A., 2003, "Vortex Method with Turbulence Sub-Grid Scale Modeling" J. of the Braz. Soc. of Mech. Sci. \& Eng., Vol. XXV, No 1, April-June, pp. 140-146.

Alcântara Pereira, L.A., Hirata, M.H. and Manzanares Filho, N., 2004, "Wake and Aerodynamics Loads in Multiple Bodies - Application to Turbo Machinery Blade Rows", J. of Wind Engineering and Industrial Aerodynamics, 92, pp. 477-491.

Bearman, P.W., 1984, "Vortex Shedding from Oscillating Bluff Bodies", Annu. Rev. Fluid Mech., 16, pp. 195-222.

Blackburn, H.M. and Henderson, R.D., 1999, "A Study of Twodimensional Flow Past an Oscillating Cylinder", J. Fluid Mech., 385, pp. 255-286.

Blevins, R.D., 1984, “Applied fluid dynamics handbook”, Van Nostrand Reinhold Co.

Blevins, R.D., 1990, "Flow Induced Vibrations", New York, Van Nostrand Reinhold.

Chorin, A.J., 1973, "Numerical Study of Slightly Viscous Flow", Journal of Fluid Mechanics, 57, pp. 785-796.

Cottet, G.H. and Koumoutsakos, P., 2000, "Vortex Methods: Theory and Practice", Cambridge University Press.

Meneghini, J.R. and Bearman, P.W., 1995, "Numerical simulation of high amplitude oscillatory flow about a circular cylinder", J. Fluid Struct., 9, pp. 435-455.

Moura, W.H., Silva, H., Alcântara Pereira, L.A. and Hirata, M.H., 2006, "Analysis of the Flow around an Oscillating Circular Cylinder in Ground Effect", ENCIT 2006 - 11th Brazilian Congress of Thermal Sciences and Engineering, Paper CIT06-0361, Curitiba, Paraná, Brazil.

Moura, W.H., 2007, "Analysis of the Flow around an Oscillating Body in the Presence of a Ground Plane" M.Sc. Dissertation, Mechanical Engineering Institute, UNIFEI, Itajubá, MG, Brasil (in Portuguese).

Mustto, A.A., Hirata, M.H. and Bodstein, G.C.R., 1998, "Discrete Vortex Method Simulation of the Flow around a Circular Cylinder with and without Rotation", A.I.A.A. Paper 98-2409, Proceedings of the 16th A.I.A.A. Applied Aerodynamics Conference, Albuquerque, NM, USA, June.

Recicar, J.N., Alcântara Pereira, L.A. and Hirata, M.H., 2006, "Harmonic Oscillations of a Circular Cylinder Moving with Constant Velocity in a Quiescent Fluid", ENCIT 2006 - 11th Brazilian Congress of Thermal Sciences and Engineering, Paper CIT06-0362, Curitiba, Paraná, Brazil.

Recicar, J.N., 2007, "High Amplitude Oscillations of a Constant Velocity Moving Body", M.Sc. Dissertation, Mechanical Engineering Institute, UNIFEI, Itajubá, MG, Brazil (in Portuguese).

Ricci, J.E.R., 2002, "Numerical Simulation of the Flow Around a Body in the Vicinity of a Plane Using Vortex Method", PhD Thesis, Mechanical Engineering Institute, UNIFEI, Itajubá, MG, Brazil (in Portuguese).

Sarpkaya, T., 1979, "Vortex-Induced Oscillations", ASME J. Appl. Mech., 46, pp. 241-258.

Sarpkaya, T., 1989, "Computational Methods with Vortices - The 1988 Freeman Scholar Lecture”, Journal of Fluids Engineering, 111, pp. 5-52.

Silva, H., 2004, "Analysis of the Flow around an Oscillating Body witch Moves with Constant Velocity", M.Sc. Dissertation, Mechanical Engineering Institute, UNIFEI, Itajubá, MG, Brazil (in Portuguese).

Shintani, M. and Akamatsu, T., 1994, "Investigation of Two Dimensional Discrete Vortex Method with Viscous Diffusion Model", Computational Fluid Dynamics Journal, 3, 2, pp. 237-254.

Stock, M.J., 2007, "Summary of Vortex Methods Literature (A lifting document rife with opnion)", April 18.

Uhlman, J.S., 1992, "An Integral Equation Formulation of the Equation of an Incompressible Fluid", Naval Undersea Warfare Center, T.R., pp. 10-086.

Williamson, C.H.K. and Roshko, A., 1988, "Vortex Formation in the Wake of an Oscillating Cylinder", J. Fluids and Structures, 2, pp. 355-381.

Williamson, C.H.K. and Govardhan, R., 2004, "Vortex Induced Vibrations", Annu. Rev. Fluid Mech., 36, pp. 413-455.
Dalton, C. and Chantranuvatana, B., 1980, "Pressure Distributions Around Circular Cylinders in Oscillating Flow", J. Fluids Engineering, 102, pp.191-195.

Kamemoto, K., 1993, "Procedure to Estimate Unstead Pressure Distribution for Vortex Method" (In Japanese), Trans. Jpn. Soc. Mech. Eng., 59:568 B, pp. 3708-3713.

Kamemoto, K., 1994, "Development of the Vortex Methods for GridFree Lagrangian Direct Numerical Simulation", Proc. Third JSME-KSME, Sendai, Japan, pp. 542-547.

Katz, J. and Plotkin, A., 1991, "Low Speed Aerodynamics: From Wing Theory to Panel Methods", McGraw Hill, Inc.

Leonard, A., 1980, "Vortex Method for Flows Simulations", J. Comp. Phys., 37, pp. 289-335.

Lewis, R.I., 1999, "Vortex Element Methods, The Most Natural Approach to Flow Simulation - A Review of Methodology with Applications", Proc. of 1st Int. Conf. on Vortex Methods, Kobe, Nov. 4-5, pp. $1-15$.

Meneghini, J.R. and Bearman, P.W., 1995, "Numerical simulation of high amplitude oscillatory flow about a circular cylinder", J. Fluid Struct., 9, pp. 435-455.

Moura, W.H., Silva, H., Alcântara Pereira, L.A. and Hirata, M.H., 2006, "Analysis of the Flow around an Oscillating Circular Cylinder in Ground Effect", ENCIT 2006 - 11th Brazilian Congress of Thermal Sciences and Engineering, Paper CIT06-0361, Curitiba, Paraná, Brazil. 